\title{
COMPUTATIONAL MODEL FOR HIGH-SPEED MULTICOMPONENT FLOWS
}

\author{
V.E. BORISOV, O.B. FEODORITOVA, N.D. NOVIKOVA, \\ YU.G. RYKOV, V.T. ZHUKOV*
}

Keldysh Institute of Applied Mathematics, RAS. Moscow, Russia

*Corresponding author. E-mail: vic.zhukov@gmail.com

\section{DOI: 10.20948/mathmontis-2020-48-4}

Summary. The article describes a robust technology for numerical simulation of multicomponent flows in the presence of strong shock waves, for example, in the flight of a high-speed vehicle with a ramjet/scramjet engine. This takes into account the phenomena of turbulence, multicomponent diffusion and heat transfer. High-temperature phenomena that occur when moving at a high supersonic speed and are associated with dissociation and ionization of the gas environment are not considered, although they fit well into the framework of the general model of a multicomponent reacting gas. The advantage of the algorithm proposed in this article is its adaptability to both the simulation of strong shock waves and to the simulations on small scales, as well as a high potential for parallelization. Illustrative examples of simulations based on the developed method are given.

\section{INTRODUCTION}

Currently, there is a rise in a new wave of interest in the implementation of controlled flight at a high supersonic speed in the stratosphere using a ramjet/scramjet engine. The processes occurring in a ramjet/scramjet engine during high-speed flight are a complex combination of gas-dynamic phenomena, such as the development of turbulent boundary layers, their interaction with shock waves, the formation of a pseudo-shock, and phenomena associated with subsonic/supersonic combustion of fuel to create thrust. Due to the high speeds, complex and highly transient structure of the processes and the resulting complexity of problem setting and high cost of natural experiments, the ability to perform predictive numerical simulation plays an important role. At the same time, it is necessary to calculate the parameters of the external flow of a high-speed aircraft in order to correctly determine the flow characteristics at the entrance to the ramjet/scramjet tract. In addition high supersonic speeds lead to various high-temperature phenomena begin to appear, which require considering this medium as a multicomponent with the possibility of chemical reactions between components. A similar situation occurs in simulation of fuel combustion in the ramjet/scramjet tract: there are components of gas, fuel and combustion products and there is an additional energy release due to chemical reactions.

All this suggests that the predictive numerical model for the flight of a high-speed aircraft with a ramjet/scramjet engine is multi-scale and, accordingly, requires a high level of detail. The solution of such problems in a reasonable time for practice is possible only on highperformance parallel computing systems.

2010 Mathematics Subject Classification: 35Q30, 65Q10, 39A70.

Key words and Phrases: Multicomponent gas mixture, Thermal conductivity, Viscosity and Diffusion, Scramjet, Hypersonic flight. 
Accordingly, the numerical algorithm should take into account a variety of physical processes on the one hand, and not be too complex to be able to control the results responsibly on the other hand. The algorithm should also be robust and well parallelized, preferably regardless of the specific architecture of the supercomputer, for example, multiprocessor or hybrid.

The purpose of this paper is to describe an algorithm of this type for numerical simulation of multi-scale multicomponent gas dynamics problems with the possibility of reactions between components. In the world literature, there is a huge number of works devoted to the actual simulations of high-speed flight in the stratosphere using the thrust of a ramjet/scramjet, as well as verification and validation of numerical modeling. These procedures are necessary elements for creating predictive numerical models. Most of the work focuses either on the external flow around the aircraft, see, for example, $[1,2]$, or on the processes occurring in the ramjet/scramjet tract, in particular, on the propagation and stabilization of the flame front depending on the geometric features of the tract, see, for example, [3,4]. The algorithm proposed in this article is especially adaptable to both the simulation of strong shock waves and the simulations on small scales, where combustion occurs, for example, as well as it has a high parallelization potential. This is achieved by using three main components. First, the method of splitting by physical processes is used; generally convective flows and flows that have a diffusive character are split. Second, the calculation of the hyperbolic part (convective flows) uses a multi-component modification of the Godunov scheme with the exact solution of the Riemann problem. Third, for solving a subproblem involving diffusive flows (the parabolic part), the original iterative method is used, a scheme of the LI-M type, see, for example, [5]. The use of a LI-M scheme allows, instead of implicit approximation of parabolic operators, to make the transition to the next time layer using explicit Chebyshev iterations, the number of which is inversely proportional to the grid parameter and is determined without using empirical parameters. The simulation is performed with the Courant number typical for hyperbolic problems. In addition to significantly reducing the simulation time of the complete problem, this methodology is well parallelized on arbitrary unstructured grids, which distinguishes it from other methodologies based on the use of implicit schemes.

The algorithm is illustrated by an example of numerical simulation of multicomponent flow in a model ramjet/scramjet tract with injection of a hydrocarbon gas-phase fuel. As a result, a multi-scale picture of the interaction of the shock wave system with the boundary layer appears and a complex flow of a multicomponent gas mixture is formed. The problem under consideration is of real practical interest, since it prepares a natural transition to threedimensional problem statements, the simulation of real layouts, combined with the modeling of external flow.

All the algorithmic constructions described above are based on the templates of the author's package of Keldysh Institute of Applied Mathematics NOISEtte [6, 7]. As a result, the new computer code MCFL (MultiComponent FLows) inherits the high parallel efficiency inherent in the NOISEtte code. This latter code is not chosen by chance, it is among the most advanced Russian CFD codes, it includes a large set of ready-made numerical models, and there are many alternative solutions. Note that there are different approaches to creating software packages for numerical modeling, see, for example, [8].

The MCFL code is in the process of development, verification tests are performed in various tasks, starting with simple ones, see, for example, [9]. In this paper, verification is 
performed on the complex problem of simulation of multicomponent mixture flows without combustion in a model combustion chamber, for which the results of experiments and simulations using different methods are known, i.e. it is essentially a specialized benchmark. Nevertheless, despite the complexity and non-linearity of the problem, the numerical results demonstrate the refinement of approximate solutions when reducing the size of the mesh cells. The simulations were performed using supercomputer resources of Keldysh Institute of Applied Mathematics (K-100, K-10) with MPI communications between various computing nodes.

The results show that the new methodology can provide a structure of complex processes. In this case, the strategy for simulation of complex problems is to solve a series of simpler problems sequentially and perform sequential verification by establishing convergence over a set of grids and comparing numerical solutions obtained using various numerical methods.

\section{BASIC EQUATIONS OF THE MATHEMATICAL MODEL FOR A MULTICOMPONENT REACTING MIXTURE}

We will assume that there is a gas mixture consisting of a basic gas, such as air, and a set of chemically reacting components, such as components of a hydrocarbon fuel. This takes into account the phenomena of turbulence, multicomponent diffusion and heat transfer, including the interaction of these processes with chemical processes. High-temperature phenomena that occur when moving at a high supersonic speed and are associated with dissociation and ionization of the basic gas will not be considered here, although they fit well into the framework of the general model of a multicomponent reacting gas.

Simulations of gas mixture flows are based on the system of Unsteady Reynolds averaged Navier-Stokes equations with the introduction of additional terms and equations for accounting for the effects of turbulence and combustion (it is assumed to sum up the repeated indexes below; $i, j=1,2,3)$, see, for example, [10,11].

Continuity equation:

$$
\frac{\partial \rho}{\partial t}+\frac{\partial\left(\rho u_{i}\right)}{\partial x_{i}}=0
$$

Momentum conservation:

$$
\frac{\partial\left(\rho u_{i}\right)}{\partial t}+\frac{\partial\left(\rho u_{i} u_{j}\right)}{\partial x_{j}}=-\frac{\partial p^{*}}{\partial x_{i}}+\frac{\partial}{\partial x_{j}}\left[\tau_{i j}\right]
$$

Energy conservation:

$$
\frac{\partial(\rho E)}{\partial t}+\frac{\partial\left(\rho u_{j} E+u_{j} p^{*}\right)}{\partial x_{j}}=-\frac{\partial q_{j}^{T}}{\partial x_{j}}+\frac{\partial}{\partial x_{j}}\left[u_{i} \tau_{i j}\right] .
$$

Equations for describing turbulence:

$$
\frac{\partial(\rho k)}{\partial t}+\frac{\partial}{\partial x_{j}}\left(\rho k \mathrm{u}_{j}\right)=\frac{\partial}{\partial x_{j}}\left(\left(\mu+\sigma_{k} \mu_{t}\right) \frac{\partial}{\partial x_{j}} k\right)+P_{k}-\rho \beta^{*} k \omega,
$$




$$
\begin{aligned}
\frac{\partial(\rho \omega)}{\partial t}+\frac{\partial}{\partial x_{j}}\left(\rho \omega \mathrm{u}_{j}\right)= & \frac{\partial}{\partial x_{j}}\left(\left(\mu+\sigma_{\omega} \mu_{t}\right) \frac{\partial}{\partial x_{j}} \omega\right)+ \\
& \alpha \rho S^{2}-\rho \beta \omega^{2}+\left(1-F_{1}\right) 2 \rho \sigma_{\omega 2} \frac{1}{\omega} \frac{\partial \omega}{\partial x_{j}} \frac{\partial k}{\partial x_{j}} .
\end{aligned}
$$

The transfer equations of chemical components with mass fractions $Y_{m}, m=1, \ldots, N_{s p}$ have the form:

$$
\frac{\partial\left(\rho Y_{m}\right)}{\partial t}+\frac{\partial\left(\rho u_{j} Y_{m}\right)}{\partial x_{j}}=-\frac{\partial J_{j, m}}{\partial x_{j}}+\dot{\omega}_{m},
$$

and the following conditions are set

$$
\sum Y_{m}=1, \quad \sum J_{j, m}=\sum \dot{\omega}_{m}=0 .
$$

Summation in these and similar formulas is based on the number of components from $m=1$ to $m=N_{s p}$. Let's explain the notations, variables, and functions used in the equations (1) $-(5): \vec{u}=\left\{u_{i}\right\}$ is the velocity vector of the averaged flow of a gas mixture; $\rho$ is the mixture density; $p^{*}=p+2 / 3 \rho k, p$ is the thermodynamic pressure of the mixture, $k$ is the energy of turbulent pulsations, $\omega$ is the specific rate of turbulent energy dissipation; $E=e+0.5 \cdot u_{k} u_{k}+k \equiv e+k+K$ is the total energy of multicomponent flow, $e$ is specific internal energy, $K$ is the kinetic energy; $\vec{q}^{T}=\left\{q_{i}^{T}\right\}$ is vector of flow caused by temperature changes; the tensor of viscous stresses $\tau_{i j}$ is put in the form

$$
\tau_{i j}=\left(\mu+\mu_{t}\right)\left[\left(\frac{\partial u_{i}}{\partial x_{j}}+\frac{\partial u_{j}}{\partial x_{i}}\right)-\frac{2}{3} \delta_{i j} \frac{\partial u_{k}}{\partial x_{k}}\right],
$$

where $\mu$ and $\mu_{t}$ are the coefficients of the molecular and turbulent viscosity of the gas mixture respectively. Specific form of diffusion and heat fluxes $J_{j, m}, q_{j}^{T}$, as well as source term $\dot{\omega}_{m}$, will be described in the next section.

To describe turbulence, the SST Menter model is used, see [12, 13], which includes two differential equations (4) for $k$ and $\omega$. But another model can also be used. An original holistic view regarding the nature of turbulence can also be found in [14], and a view of critical phenomena from the standpoint of non-equilibrium phase transitions can be found in [15]. In addition non-traditional approach to conservation laws theory is contained in [16].

The system of equations (1) - (5) is supplemented by the Mendeleev-Clapeyron equation of state for a mixture of ideal gases

$$
p=\rho \frac{R}{M} T,
$$


where $R$ is the universal gas constant, $M$ is the molar mass of the total mixture and $1 / M=\sum Y_{m} / M_{m}, M_{m}$ is the molar mass of the $m$ th component.

For a reacting multicomponent mixture, the specific internal energy $e$ is determined via the enthalpy $h$ of the mixture using the formula $e=h-p / \rho$. At the same time

$$
h=h_{s}+h_{c h} \equiv \int_{T_{0}}^{T} C_{p}(\theta) d \theta+\sum \Delta h_{m}^{0} Y_{m}=\sum h_{m} Y_{m} \equiv \sum Y_{m} \int_{T}^{T} C_{p, m}(\theta) d \theta+\sum \Delta h_{m}^{0} Y_{m}
$$

where $C_{p, m}(T)$ is the heat capacity of the $m$ th component at constant pressure, $\Delta h_{m}^{0}$ is the enthalpy required to form $1 \mathrm{~kg}$ of the component at standard temperature $T_{0}=298.15 \mathrm{~K}$.

Finally, we note that instead of the energy equation (3), we often use the equation for enthalpy, which is a consequence of (1) - (3). The advantage of the equation for enthalpy is that it describes the contribution of the chemical energy of reactions to the increase in the flow temperature.

\section{COEFFICIENTS OF MULTICOMPONENT DIFFUSION, VISCOSITY, THERMAL CONDUCTIVITY, AND CHEMICAL REACTIONS}

In the framework of the model considered in this paper, the flux $\vec{q}^{T}$ consists of two parts: the actual heat flux and the diffusion flux of the mixture:

$$
q_{j}^{T}=-\lambda \frac{\partial T}{\partial x_{j}}+\sum h_{m} J_{m, j} .
$$

Diffusion flux $J_{m, j}$ of each component is defined according to the formula $J_{m, j}=-\rho D_{m} \cdot \partial Y_{m} / \partial x_{j}$, where the effective diffusion coefficients $D_{m}$ can be found by Wilke's rule, see [17-19], using binary diffusion coefficients of the individual components. The coefficients of the mixture's viscosity and thermal conductivity can be written using, for example, the Wilke approximations [17] and the Mason-Saxena approximations [20]. A more accurate approximation according to [21] of the effective coefficients of viscosity, thermal conductivity, and diffusion is written using the so called reduced Q-integrals as the first approximations of the Chapman-Enskog method.

We will use simpler approximations at this stage. To calculate the molecular viscosity of each mixture component, the Sutherland formula is used $\mu_{m}(T)=C_{1, m} T^{\Delta_{m}} /\left(T+C_{0, m}\right)$ with specially chosen constants. The thermal conductivity and diffusion coefficients of each component are taken as $\lambda_{m}=C_{p, m}(T) \mu_{m} / \operatorname{Pr}$ and $D_{m}=\mu_{m} /(\rho S c)$ assuming Prandtl number $\operatorname{Pr}$ and Schmidt number $S c$. To average the coefficients of the thermal conductivity and the viscosity, we use the following formulas

$$
\mu=\frac{1}{2}\left[\sum \mu_{k} X_{k}+\left(\sum\left(X_{k} / \mu_{k}\right)\right)^{-1}\right], \quad \lambda=\frac{1}{2}\left[\sum \lambda_{k} X_{k}+\left(\sum\left(X_{k} / \lambda_{k}\right)\right)^{-1}\right],
$$

where $X_{k}=Y_{k} M / M_{k}$. The mixture turbulent viscosity $\mu_{t}$ when using Menter model (4) is taken according to the formula $\mu_{t}=\rho k / \omega$. 
The term responsible for chemical reactions is described in the standard way

$$
\dot{\omega}_{m}=M_{m} \sum_{j=1}^{N_{r}} \dot{\omega}_{m j}, \quad \dot{\omega}_{m j}=\left(v_{m j}^{\prime \prime}-v_{m j}^{\prime}\right)\left(k_{f j} \prod_{l=1}^{N_{s p}}\left[\frac{\rho Y_{l}}{M_{l}}\right]^{v_{l j}^{\prime}}-k_{b j} \prod_{l=1}^{N_{s p}}\left[\frac{\rho Y_{l}}{M_{l}}\right]^{v_{l j}^{\prime \prime}}\right) \text {. }
$$

Here $N_{r}$ is the number of reactions, $v_{m j}^{\prime}, v_{m j}^{\prime \prime}$ are the molar stoichiometric coefficients, $k_{f j}, k_{b j}$ are respectively, the forward and backward reaction rate coefficients, depending on the temperature.

\section{MAIN FEATURES OF THE NUMERICAL ALGORITHM}

Let us introduce the state vector of conservative variables $\boldsymbol{U} \equiv \rho\left(1, u_{1}, u_{2}, u_{3}, E, k, \omega,\left\{Y_{m}, m=1, \ldots, N_{s p}\right\}\right)$. Then the difference approximation of the system of equations (1) - (5) can be written as follows

$$
\frac{\partial}{\partial t} \boldsymbol{U}+C_{h}(\boldsymbol{U})=D_{h}(\boldsymbol{U})
$$

where $C_{h}(\boldsymbol{U})$ is nonlinear convective difference operator on a grid with a characteristic cell diameter $h$, and $D_{h}(\boldsymbol{U})$ is, generally speaking, a nonlinear diffusive operator. By linearizing (11) and taking a time step $\tau$, an explicit scheme can be written in the following form

$$
\frac{\boldsymbol{U}_{j+1}-\boldsymbol{U}_{j}}{\tau}+\tilde{C}_{h} \boldsymbol{U}_{j}=\tilde{D}_{h} \boldsymbol{U}_{j} .
$$

The main goal is to offer a specific implementation of (11) that preserves the time step constraints that correspond only to the hyperbolic part. To achieve this goal, we use the method of splitting the system into hyperbolic and diffusion parts and solving the appropriate diffusion problem using a special iterative process. Namely, we will solve equations (11) at each time step using two stages. At the first stage we put $\tilde{D}_{h} \equiv 0$ and find the solution of an intermediate difference hyperbolic equations using an explicit scheme

$$
\frac{\overline{\boldsymbol{U}}_{j+1}-\boldsymbol{U}_{j}}{\tau}+\tilde{\boldsymbol{C}}_{h} \boldsymbol{U}_{j}=0 \text {. }
$$

In this case, the Godunov method for calculating flows with an exact solution of the Riemann problem for a multicomponent mixture is used [22]. Note that the Riemann problem for turbulent equations (4) is solved separately. Using an exact solution to the Riemann problem allows us to better take into account the specifics of the flows with strong shock waves. As is well known, multicomponent flow generates a number of computational instabilities, to mitigate which the Godunov scheme must be modified. The modification [22] uses the so-called "double flow" method, see, for example, [23].Thus, intermediate values $\overline{\boldsymbol{V}}_{j}, \overline{\boldsymbol{U}}_{j}=\rho \overline{\boldsymbol{V}}_{j}$ are obtained at the hyperbolic stage. At the second stage we put $\tilde{C}_{h} \equiv 0$ and write difference parabolic problem with new initial data 


$$
\frac{\boldsymbol{U}_{j+1}-\overline{\boldsymbol{U}}_{j}}{\tau}=\tilde{D}_{h} \hat{\boldsymbol{U}}_{j} .
$$

If in (14) $\boldsymbol{U}_{j}$ is taken instead of $\hat{\boldsymbol{U}}_{j}$, then the summation of (14) and (13) leads to the conservation equations (12), but the resulting scheme requires diffusive limitation on the time step size, $\tau \sim h^{2}$. To avoid such a strict limitation we find $\hat{\boldsymbol{U}}_{j}$ in the course of iterations of the LI-M scheme detailed in [5]. The construction of this scheme is based on the use of properties of Chebyshev polynomials. Approximation and stability are inherent in the construction of the scheme. The equations (14) are the result of final iteration of the LI-M scheme, and the number of iterations $p$ is inversely proportional to the grid parameter $h$ and is determined without using empirical parameters by an exact formula $p=\left\lceil 0.25 \pi \sqrt{\tau \lambda_{\max }+1}\right\rceil$, where $\lambda_{\max }$ is the upper bound of the spectrum of the discrte operator $\tilde{D}_{h}$. The resulting algorithm remains stable under the hyperbolic time step constraint $\tau \sim$ const $\cdot h$ and lends itself well to the parallelization process.

Above it is described only the principle scheme of splitting. In the concrete implementation at the current stage of development the turbulent equations (4) do not participate.

\section{SIMULATION RESULTS}

Below there are demonstrative numerical simulation results of multicomponent turbulent flows with strong shock waves performed on a multiprocessor supercomputer K-100 of the hybrid architecture using the methodology described above. The simulation strategy is developed using the example of a two-dimensional problem of modeling the flow of a chemically reacting multicomponent medium in a channel with a backward step. This channel is a model of the combustion chamber of a high-speed aircraft. The task is set as follows, see [24]. The combustion chamber is a duct with a step that consists of a short part with an expansion angle of $0.5^{\circ}$ and a long constant-area part, Figure 1.

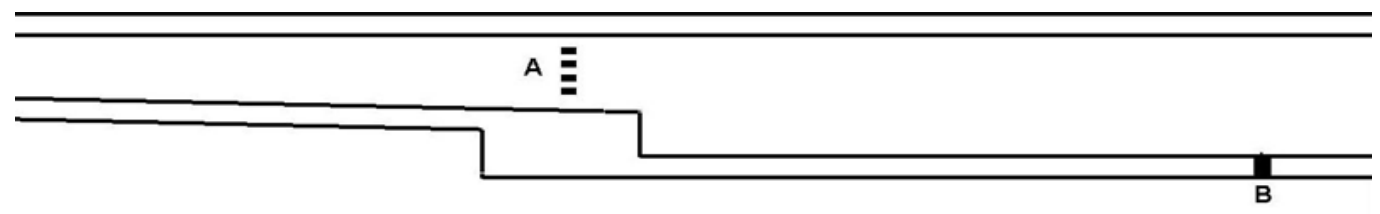

Figure 1. The schematic of the combustion chamber

At the entrance to the chamber, inflow supersonic flow (Mach number 2.5) is set, containing the products of hydrocarbon fuel combustion. The fuel injectors are located in the front of the chamber in the area marked with the letter A. To ensure the ignition of the mixture, a short-term locking of the channel with a stream of compressed air is used, the supply of which is carried out across the main flow using a pneumatic throttle B. Calculations are performed on a refined sequence of nested meshes, indicated below 1x, 2x, 4x. Each of the meshes is obtained from the previous one by doubling the number of cells in both directions. The $1 \mathrm{x}$ grid consists of $\sim 110$ thousand nodes, the $2 \mathrm{x}$ grid $\sim 440$ nodes, etc. In [25], similar numerical experiments were performed using the OpenFOAM software package. Note 
that the results of calculations on the $2 \mathrm{x}$ and $4 \mathrm{x}$ grids do not visually differ much, so the graphs below are given for the $1 \mathrm{x}$ and $2 \mathrm{x}$ grids. The time-marching scheme is implemented to obtain a steady-state solution or oscillating mode in the combustion process in the chamber. The second order spatial Galerkin approximation is used in the NOISEtte for all viscous, heat conduction and diffusion fluxes.

In Figure 2 the flow structure of a multicomponent mixture is shown. Figure 3 shows the flow details in the fuel injection zone in the gas phase, and in additional, two coordinate lines $x=$ const and $y=$ const are marked, along which Figure 4 shows one-dimensional profiles of fuel concentration and pressure for grids $1 \mathrm{x}$ and 2x. Figures 2 and 4 demonstrate the convergence of approximate solutions when reducing the size of grid's cells.

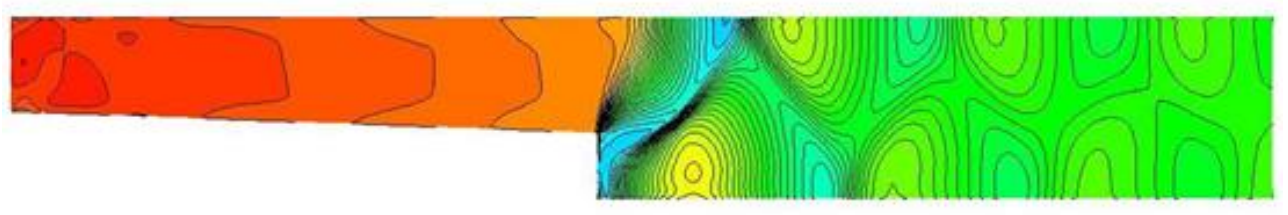

$\mathrm{b}$

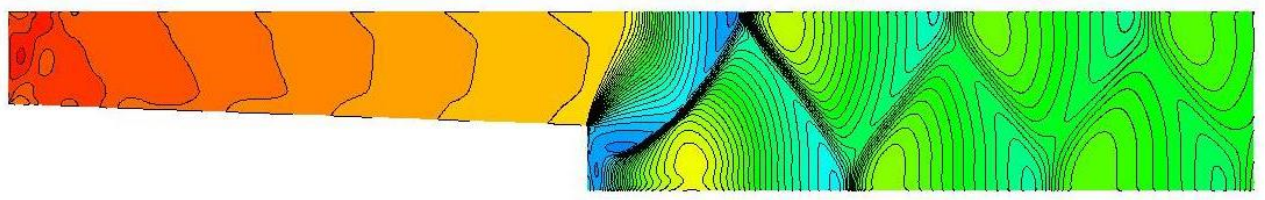

Figure 2. Normalized pressure field on the grids $1 \mathrm{x}$ (a) and $2 \mathrm{x}(\mathrm{b})$

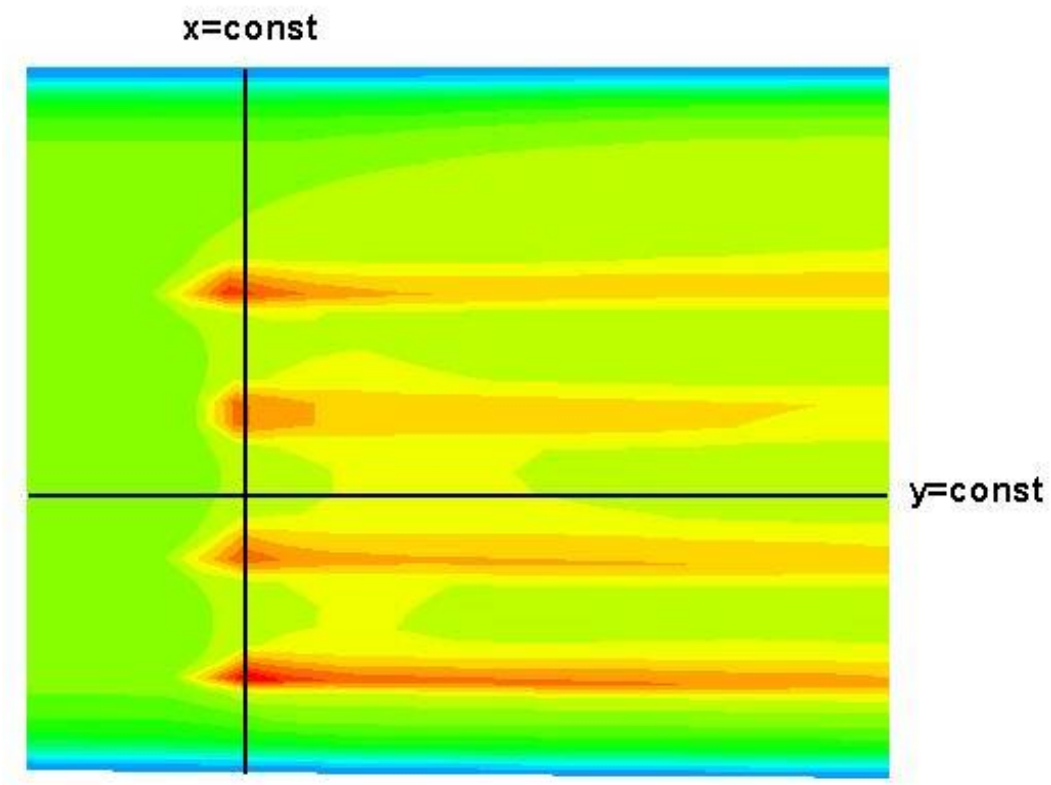

Figure 3. Fuel injection zone; lines $x=$ const and $y=$ const for the imaging 1D profiles 

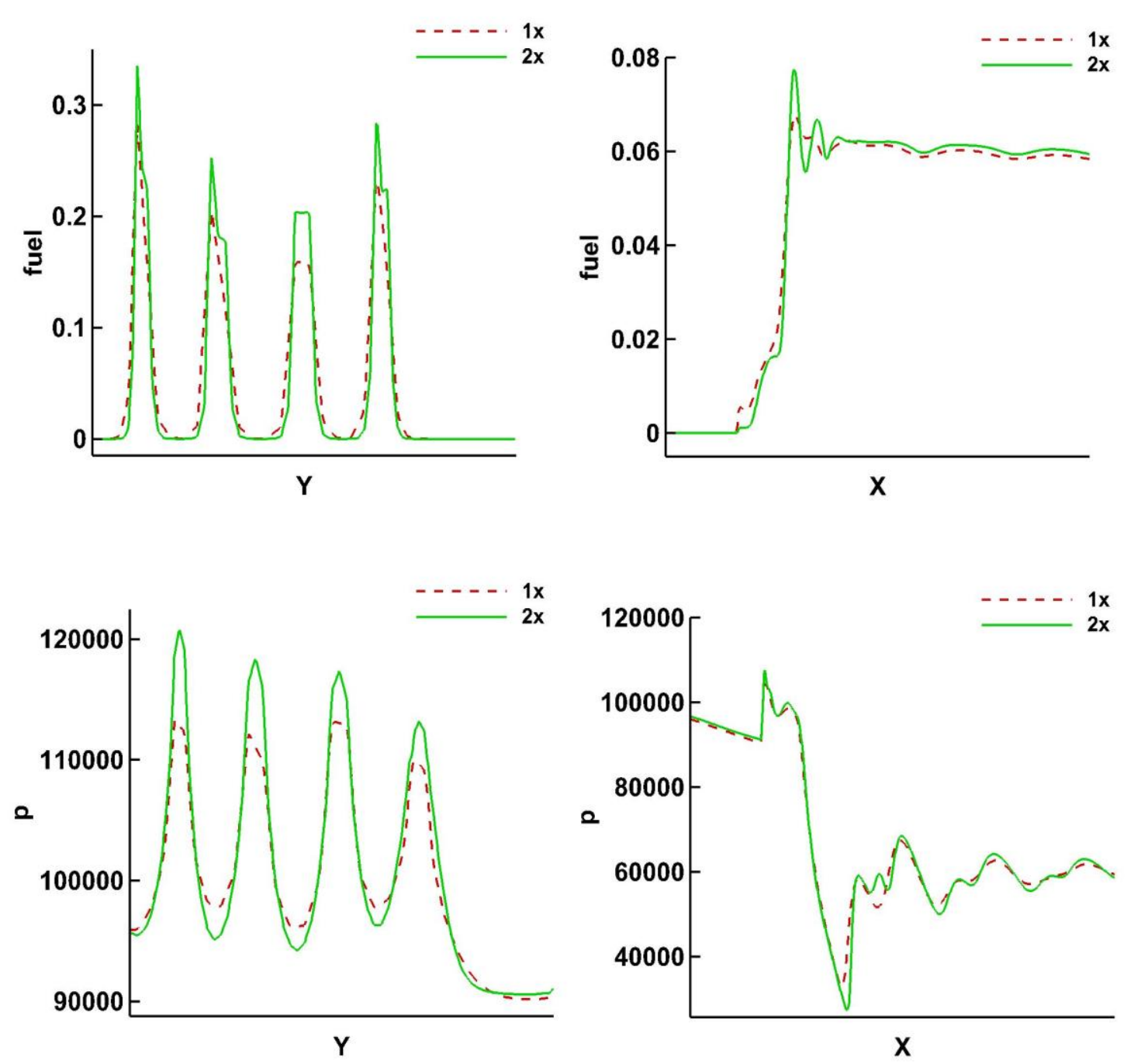

Figure 4. Profiles of fuel fraction (top row) and pressure (bottom row) along the chosen lines for the grids $1 \mathrm{x}$ and $2 \mathrm{x}$

Majority of numerical-experimental investigations of the flow in a chamber with the step were implemented for the validation of numerical technologies, see for example [24]. Similarly, the results presented here show that the developed numerical technique describes high-speed flow in the combustor duct correctly.

\section{CONCLUSION}

The paper presents an original numerical method for integrating multi-component NavierStokes equations. The difference method used allows us to more accurately account for the presence of strong shock waves and maintain stability under hyperbolic restrictions on the value of the time step. The results of simulations are qualitatively consistent with experimental data and calculated data from other studies. 
The proposed algorithm is well parallelized, which makes it a robust tool for numerical modeling complex flows on grids with a large number of nodes, for example, the external flow of a high-speed aircraft and the internal flow in a ramjet/scramjet tract. The presented methodology can serve as a development basis for creating a fully functional tool for numerical modeling of complex processes on high-performance computing systems.

Acknowledgements: This work was supported by Russian Science Foundation, project № 19-71-30004.

\section{REFERENCES}

[1] T. Murugan, S. De, V. Thiagarajan, "Validation of three-dimensional simulation of flow through hypersonic air-breathing engine", Defence Science Journal, 65(4), 272-278 (2015).

[2] T.V. Markova et all, "Simulating flow around scaled model of a supersonic vehicle in wind tunnel”, J. Phys.: Conf. Ser., 774, 012095 (2016).

[3] F.H.E. Ribeiro, R. Boukharfane, A. Mura, "Highly-resolved large-eddy simulations of combustion stabilization in a scramjet engine model with cavity flameholder", Computers and Fluids, 197, 104344 (2020).

[4] E.Jeong, S.O'Byrne, I-S. Jeung, A.F.P. Houwing, "The effect of fuel injection location on supersonic hydrogen combustion in a cavity-based model scramjet combustor", Energies, 13(1), $1-16(2020)$.

[5] V.T. Zhukov, "On explicit methods for the time integration of parabolic equations", Math. Models Comput. Simul., 3(3), 311-332 (2011).

[6] P.A. Bakhvalov, I.V. Abalakin, T.K. Kozubskaya, "Edge-based reconstruction schemes for unstructured tetrahedral meshes", Int. J. Numer. Methods Fluids. 81(6), 331-356 (2016).

[7] I.V. Abalakin. A.V. Gorobets, A.P. Duben, T.K. Kozubskaya, P.A. Bakhvalov, "Parallel Research Code NOISEtte for Large-Scale CFD and CAA Simulations", Numerical methods and programming. 13, 110-125 (2012).

[8] I.N. Konshin, K.M. Terekhov, Yu.V. Vassilevski, "Numerical modelling via INMOST software platform”, Mathematica Montisnigri, 47, 75-86 (2020).

[9] V.T. Zhukov, N.D. Novikova, O.B Feodoritova, "An Approach to Time Integration of the NavierStokes Equations", Comp. Math. and Math. Physics, 60(2), 272-285 (2020).

[10] T. Poinsot, D. Veynante, "Theoretical and numerical combustion", Edwards, 3rd Edition, 522 p. (2011).

[11] S.T. Surzhikov, “Radiation gas dynamics of the spacecraft. Multi-temperature models”, IPMech RAS, 706 p. (2013) (in Russian).

[12] F.R. Menter, "Two-equation eddy-viscosity turbulence models for engineering applications", AIAA-Journal, 32(8), 269-289, (1994).

[13] W. Vieser, T. Esch, F. Menter. "Heat transfer predictions using advanced two-equation turbulence models", CFX Validation Report 10/0602, AEA Technology, 1-69 (2002).

[14] M.Ya. Marov, A.V. Kolesnichenko, "Turbulence and self-organization", Springer, (2013), 657 p.

[15] E.V. Radkevich, E.A. Lukushov, N.N. Yakovlev, O.A. Vasilieva, M.I. Sidorov, "Introduction to the generalized theory of non-equilibrium phase transitions and thermodynamic analysis of solid environment mechanics tasks", Lomonosov Moscow State University, (2019), 342 c. (in Russian)

[16] Yu.G. Rykov, "Extremal properties of the functionals connected with the systems of conservation laws", Mathematica Montisnigri, 46, 21-30 (2019).

[17] C.R. Wilke, "A viscosity equation for gas mixtures", J. Chem. Phys., 18(4), 517-522 (1950).

[18] C.R. Wilke, "Diffusional Properties of Multicomponent Gases", Chemical engineering progress, 46(2), 95-104 (1950).

[19] D. F. Fairbanks, C.R. Wilke, "Diffusion Coefficients in Multicomponent Gas Mixtures", Ind. 
Eng. Chem., 42(3), 471-475 (1950).

[20] E.A. Mason, S.C. Saxena, "Approximate Formula for the Thermal Conductivity of Gas Mixtures", Physics of Fluids, 1(5), 361-369 (1958).

[21] J.H. Ferziger, H.G. Kaper,"Mathematical Theory of Transport Processes in Gases", North-Holland Publishing Company, 1972.

[22] V.E. Borisov, Yu.G. Rykov, "Modified Godunov method for multicomponent flow simulation", J. Phys.: Conf. Ser., 1250 (2019), 012006.

[23] G. Billet, R. Abgrall, "An Adaptive Shock-Capturing Algorithm for Solving Unsteady Reactive Flows", Computers \& Fluids, 32 (10), 1473-1495 (2003).

[24] M. Ivankin, A. Nikolaev, V. Sabelnikov, A. Shiryaeva, V. Talyzin, V. Vlasenko, "Complex Numerical-Experimental Investigations of Combustion in Model High-Speed Combustor Ducts", Acta Astronaut., 158, 425-437(2019).

[25] V.T. Zhukov, N.D. Novikova, O.B Feodoritova, "On the Numerical Simulation of Combustion in a Scramjet Combustor Using OpenFOAM”, Math Models Comput Simul, 11(2), 266-276 (2019).

Received June 6, 2019 